\title{
Investigation of Hydrogen-Defect Interaction in Tungsten by the Probe
}

\section{Fluence Method}

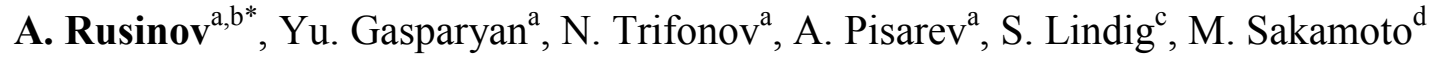 \\ ${ }^{a}$ National Research Nuclear University "MEPHI”, Kashirskoe sh. 31., Moscow, 115409, Russia, \\ ${ }^{b}$ Interdisciplinary Graduate School of Engineering Sciences, Kyushu University, Kasuga,,816-8580, \\ Japan
}

${ }^{c}$ Max-Planck-Institut für Plasmaphysik, EURATOM Association, Boltzmanstr.2, D-85748 Garching,

Germany

${ }^{d}$ Advanced Fusion Research Institute for Applied Mechanics, Kyushu University, Kasuga, Fukuoka, 816-8580, Japan

\begin{abstract}
Deuterium trapping by defects in $\mathrm{W}$ polycrystalline foils during ion bombardment was investigated by thermal desorption spectroscopy using the low fluence probe method. Probe TDS spectra showed, that there are at least 6 peaks in the region $350-900 \mathrm{~K}$ : at $370 \mathrm{~K}, 450 \mathrm{~K}$, $530 \mathrm{~K}, 580 \mathrm{~K}, 630 \mathrm{~K}, 750 \mathrm{~K}$. Experiments with as received samples showed that D is trapped mainly in low energy traps in the region 390-650 K. These defects are attributed to technological defects such as dislocations and vacancies. The peak at $750 \mathrm{~K}$ easily disappears when annealing the sample at about $1300 \mathrm{~K}$ and can be attributed to vacancy clusters. The peak at $630 \mathrm{~K}$ irreversibly increases at high fluence, it also appears when annealing the sample above $1300 \mathrm{~K}$. This peak can be attributed to voids. Voids of about $20 \mathrm{~nm}$ in diameter in the near surface region were observed by FIB/SEM in cases when $630 \mathrm{~K}$ peak was observed.
\end{abstract}

JNM keywords: I0400, F0800, R0200, T1000

PSI-19 keywords: Deuterium implantation, Defects, Thermal desorption, Tungsten 
PACS: 68.43.Vx, 61.72.Qq, 61.80.Jh, 61.72.J

Corresponding author address:

*Corresponding author e-mail: rusinov.aleksandr@gmail.com

Presenting author: Rusinov Aleksandr

Presenting author e-mail: rusinov.aleksandr@gmail.com 


\section{Introduction}

Tungsten is a candidate for the divertor plates in the future thermonuclear reactor. Though solubility of hydrogen isotopes in tungsten is rather low, tritium can be accumulated in radiation defects that will increase problems with radiation safety.

Thermal desorption spectroscopy (TDS) is often used to investigate hydrogen-defect interaction. Deuterium release in TDS from tungsten was studied by many researchers as overviews $[\mathbf{1 , 2}]$ demonstrate. Ion implantation is commonly used in these investigations, for example [3-10]. Ions produce radiation defects, which interact with implanted deuterons, so TDS give information about deuterium-defect interaction. The more refined method of probe low fluence implantation is based on the expectation that the number of radiation defects produced by small number of probe ions is much less than the number of defects pre-existing to this low probe fluence. Therefore TDS after probe fluence give information about defects that existed in the sample before probe TDS analyses. This method was used, for example, in $[3,4]$.

This paper describes probe low fluence TDS experiments with W samples, which were foils of different pre-histories (either as-received, or annealed, or irradiated, or irradiated and then annealed.)

\section{Experimental}

Experiments were performed in MEDION ion beam facility (MEPHI, Moscow). The ion beam was extracted from a duoplasmatron ion source, separated by a magnet, and directed onto the sample through a $3 \mathrm{~mm}$ diaphragm. Ions $\mathrm{D}_{3}{ }^{+}$with the energy of $10 \mathrm{keV} / \mathrm{D}_{3}{ }^{+}$and the flux of about $10^{14} \mathrm{D} / \mathrm{cm}^{2} \mathrm{sec}$ were used for two purposes: for radiation damage (up to $\left.5 \times 10^{18} \mathrm{D} / \mathrm{cm}^{2}\right)$ and for probe low fluence $\left(2 \times 10^{16} \mathrm{D} / \mathrm{cm}^{2}\right)$ TDS measurements. We will use the term "irradiation" for the former experimental step and the term "implantation" for the 
latter step. Both irradiation and implantation were performed at room temperature. Samples with natural defects were also analyzed by the probe TDS.

The sample $15 \times 60 \mathrm{~mm}$ in size, cut from a $50 \mu \mathrm{m}$ polycrystalline tungsten foil produced by Plansee was mounted on two water cooled feedthroughs. The sample could be heated resistively up to $1750 \mathrm{~K}$ with the linear ramp of $2 \mathrm{~K} / \mathrm{sec}$ for TDS measurements, which were performed in 10 min after ion implantation. The temperature near the beam strike area was measured by a spot welded thermocouple.

Release of 20 gases was monitored simultaneously by a quadrupole mass-spectrometer (QMS). The absolute sensitivity of QMS to He was routinely measured using a helium leak after every experiment. Relative sensitivities for $\mathrm{D}_{2}$ and $\mathrm{He}$ was determined in separate experiment using a system including two leak valves and baratron (procedure is identical to [11]).

Samples used were either "as received" (without any treatment) or "recrystallized" (annealed at $1750 \mathrm{~K}$ for 1 hour) or "radiation damaged" (after radiation, but not annealed) or "annealed after radiation damage". Grains in "as-received" sample were elongated parallel to the surface with the size of $1-5 \mu \mathrm{m}$, while grains in "recrystallized" sample were $10-50 \mu \mathrm{m}$ in size and symmetrical in all dimensions (see also [12]). The recrystallized samples demonstrated a very good reproducibility of TDS spectra. If not mentioned specially, the sample was routinely annealed at $1750 \mathrm{~K}$ for 1 hour after every TDS measurement to obtain a good reproducibility of TDS measurements.

After experiments, the sample was removed, cut by a focused ion beam (FIB) and the cross-section was analyzed by scanning electron microscopy (SEM) at Max-Planck-Institut für Plasmaphysik (Garching, Germany).

\section{Results and discussion}


Ion irradiation leads to development of the defect structure. Therefore, TDS measured just after ion irradiation vary with ion fluence. As an example, Figure 1 shows TDS obtained from well annealed (recrystallized) sample irradiated by the fluence from $2.5 \times 10^{16}$ to $2 \times 10^{18} \mathrm{D} / \mathrm{cm}^{2}$. Release of ion implanted deuterium was observed in the temperature range of 390-900 K. There are three well seen maxima in the spectra at about $400 \mathrm{~K}, 570 \mathrm{~K}$, and $750 \mathrm{~K}$. All amplitudes increase with the fluence. The peaks are wide and may be formed by overlapping of more narrow peaks. The peak at $750 \mathrm{~K}$ appears at the fluence higher than $10^{17} \mathrm{D} / \mathrm{cm}^{2}$. Increase of fluence leads to increase of concentrations of all defects and appearance of new types of defects.

Heating of the sample after TDS run at $1750 \mathrm{~K}$ for 1 hour leads to almost complete annealing of accumulated defects. This annealing was used after every TDS measurement shown in Figure 1. If samples are heated for a shorter time, defects can not disappear completely, but their concentration gradually decreases in a series of short heatings at $1750 \mathrm{~K}$. This is demonstrated in Figure 2. The recrystallized sample was irradiated to the fluence of $6 \times 10^{17} \mathrm{D} / \mathrm{m}^{2}$ and annealed to $1750 \mathrm{~K}$ just for a few minutes. Then, a series of probe TDS (probe fluence $2 \times 10^{16} \mathrm{D} / \mathrm{cm}^{2}$ ) was made. The sample was kept at the maximum temperature $1750 \mathrm{~K}$ only for a few minutes after each probe TDS run in this series of experiments. One can see that the peak amplitudes decrease gradually from one probe TDS to another. Thus, during probe TDS heating, the defects created by initial high fluence irradiation gradually annealed in the series of probe runs. After the fourth probe implantation, the peak $630 \mathrm{~K}$ stabilized at a steady state level, while other amplitudes continued to decrease. Finally, the sample was well annealed after several heating cycles, and the respective probe TDS demonstrated the spectrum with two wide peaks (390 K and $630 \mathrm{~K}$ ). 
One must mention from these series, that there are at least 5 TDS peaks in the region of 390-700 K: $390 \mathrm{~K}, 450 \mathrm{~K}, 530 \mathrm{~K}, 580 \mathrm{~K}, 630 \mathrm{~K}$. Two of them appear as shoulders of the neighbor peaks (they can be identified also from Figure 5).

One must mention that there is no peak $750 \mathrm{~K}$ in Figure 2, though this peak is seen in Figure 1 at irradiation to the fluence higher than $10^{17} \mathrm{D} / \mathrm{cm}^{2}$. One can suppose that defects responsible for this peak are easily annealed in the first TDS run made just after irradiation, when the sample was kept at $1750 \mathrm{~K}$ for a very short time. Only these defects disappear after the first TDS measurement; other defects are annealed much slower as it follows from Figure 2.

The third series of experiments shown in Figure 3 demonstrates influence of temperature on annealing of ion induced defects. As in the previous series, the sample was first irradiated to $6 \times 10^{17} \mathrm{D} / \mathrm{cm}^{2}$ and then annealed not to $1750 \mathrm{~K}$, as usually, but only to $873 \mathrm{~K}$. In this case, all D was removed from the sample (as TDS given in Figure 1 demonstrates), but many defects remained not annealed. In the first probe TDS, the sample was heated only to $873 \mathrm{~K}$ and kept at this temperature for $30 \mathrm{~min}$. Then, a series of probe runs with heating to $1073 \mathrm{~K}(\mathrm{~b}), 1273 \mathrm{~K}$ (c), $1473 \mathrm{~K}$ (d), $1623 \mathrm{~K}$ (e), $1750 \mathrm{~K}$ (f), and again $1750 \mathrm{~K}$ (g) was performed. The sample was kept at the respective maximum temperatures for $30 \mathrm{~min}$ after runs (a)-(e) and 1 hour after run (f). That is, before run (a), the sample was only shortly annealed at $873 \mathrm{~K}$, but before runs (b-f) it was annealed for longer time at $\mathrm{T}_{\max }=873,1073$, $1273,1473,1623$, and $1750 \mathrm{~K}$.

The first probe TDS (curve (a) in Figure 3) shows the $750 \mathrm{~K}$ peak and this peak is relatively high in Figure 3 (contrary to Figure 2 where this peak disappears after heating to $1750 \mathrm{~K}$ ). Annealing at $873 \mathrm{~K}$ in the first probe run (a) before the second probe run (b) led to a small decrease of concentrations of all defects and respective decrease of all the peaks in the spectrum (b). It was observed, that the amplitude of the peak $750 \mathrm{~K}$ decreases with increase of 
$\mathrm{T}_{\max }$ (Figure 3). Annealing at $\mathrm{T}_{\max }=1273 \mathrm{~K}$ in run (c) led to complete disappearance of peak $750 \mathrm{~K}$ in the next run (d). These experiments explain why the defects, which correspond to peak $750 \mathrm{~K}$, disappear in routine TDS procedure with heating up to $1750 \mathrm{~K}$ in the probe TDS in Figure 2.

It was observed in experiment [4] that peak near $750 \mathrm{~K}$ appears at an elevated fluence and this appearance correlates with appearance of small sized vacancy clusters deduced from positron annihilation measurements. These vacancy clusters can disappear either due to dissociation or agglomeration in larger clusters during heating the sample to high temperature. The effect of disappearance of small clusters can explain some observations from our experiments. Annealing at $1273 \mathrm{~K}$ (Figure 3, curve d) leads to disappearance of peak $750 \mathrm{~K}$ and pronounced increase of the low temperature part. This could be due to re-arrangement of defects: small clusters transform into voids and single vacancies.

Another set of experiment was performed with as received sample, which was neither annealed nor ion damaged to investigate interaction of deuterons with natural defects. Five performed probe TDS are shown in Figure 4. These probe TDS were made to the final temperatures $\mathrm{T}_{\max }=900(\mathrm{a}), 1090$ (b), 1500 (c), 1750 (d), and $1750 \mathrm{~K}(\mathrm{e})$, and the annealing time at these temperatures was $30 \mathrm{~min}$ for (a-d) and $60 \mathrm{~min}$ for (e). So, the first TDS (a) was measured for as received sample, while the second to fifth probe experiments were made with the sample, which was pre-annealed at temperatures 900 (b), 1090 (c), 1500 (d), and $1750 \mathrm{~K}$ (e), respectively. The first probe TDS (Figure 4) shows presence of low temperature peaks, which are higher than that in the well annealed sample (compare to the probe TDS of the annealed sample in Figure 2). Weak defects at $390 \mathrm{~K}$ can be attributed to various trapping sites, such as dislocations, surface impurities [10], as well as grain boundaries, which concentration in the as received material is much higher, than in the recrystallized one. One can see that the spectra transforms after the first probe TDS: low temperature peak increases 
at the cost of decrease of the high temperature peak. Finally, after annealing at $1750 \mathrm{~K}$ for an hour we come to the probe TDS with two visible maxima at about $370 \mathrm{~K}$ and at $520 \mathrm{~K}$ but without $630 \mathrm{~K}$ and $750 \mathrm{~K}$ peaks. This probe TDS was reproduced for many samples, which were regularly implanted by a small fluence (but not irradiated to high fluence) and were regularly annealed to $1750 \mathrm{~K}$ for an hour. The peak $630 \mathrm{~K}$ appears only if the sample has been irradiated by a high fluence as in Figure 2. If the peak $630 \mathrm{~K}$ appears once, it never disappears even after annealing at $1750 \mathrm{~K}$, so one can see it in all subsequent spectra.

One can conclude from Figure 2 and Figure 4 that the defect structure of well annealed samples without irradiation and well annealed samples with high fluence irradiation are different. Defects that give peak $630 \mathrm{~K}$ after ion irradiation are of high concentration and very stable. Defects that give intensive $630 \mathrm{~K}$ peak are accumulated with irradiation fluence. The difference from other defects is that they can not be annealed in our conditions (1 hour at $1750 \mathrm{~K}$ ). There was no peak $750 \mathrm{~K}$ in the as received sample, so defects that give this peak are of radiation nature.

Let us denote $\mathrm{F}_{\max }$ as a maximum fluence in a series of irradiations of the sample. If $\mathrm{F}_{\max }$ is large, the peak at $630 \mathrm{~K}$ in probe TDS increases irreversibly. This is shown in Figure 5 and means accumulation of this kind of defects in the sample with $\mathrm{F}_{\max }$ increase.

Samples, exposed in TDS experiments to $F_{\max }=2 \times 10^{18}$ and $5 \times 10^{18} \mathrm{D} / \mathrm{cm}^{2}$ were sectioned by FIB and analyzed by SEM. Numerous voids were observed in the near surface region (Figure 6). The voids were located mainly in a narrow region within $500 \mathrm{~nm}$ from the surface, though some voids were observed also deeper under the surface. There were no voids deeper in the bulk. The voids were observed only inside the irradiation spot, and there were no voids outside the spot. Thus, the voids are induced by ion irradiation. These voids, which are rather stable at annealing at $1750 \mathrm{~K}$ can be responsible for the peak $630 \mathrm{~K}$, which is also stable at annealing as it was discussed above. These defects are suggested to be formed from 
small vacancy clusters which migrate at high temperature during annealing. Release from voids created in tungsten by $\mathrm{MeV}$ electron or proton irradiation was observed in [6] at a similar temperature. Eleveld et al. [8] observed second desorption peak at $650 \mathrm{~K}$ in $\mathrm{W}$ that has bubbles created by He pre-irradiation. Growth of voids is possible both during irradiation to high fluence and during annealing at high temperature after irradiation to a smaller fluence due to cluster agglomeration. Once voids appear, they serve as a sink for vacancies that lead to their further growth. These voids cannot be annealed at $1750 \mathrm{~K}$.

\section{Conclusion}

Deuterium thermal desorption from ion induced and natural defects in $\mathrm{W}$ polycrystalline sheet (Plansee, $50 \mu \mathrm{m}$ ) was studied by the probe fluence method. Tungsten sample was irradiated in the range of $2 \times 10^{16}-5 \times 10^{18} \mathrm{D} / \mathrm{cm}^{2}$. Probe TDS showed that there are at least 6 peaks: $390 \mathrm{~K}, 450 \mathrm{~K}, 530 \mathrm{~K}, 580 \mathrm{~K}, 630 \mathrm{~K}$, and $750 \mathrm{~K}$. Some of them are often combined in broader peaks. Low temperature release in the range of 350-600 K was also observed in TDS from as received sample. Low temperature $(<600 \mathrm{~K})$ defects might be attributed to release from point defects, dislocations, and grain boundaries. Peak $750 \mathrm{~K}$ appears at large fluence; and defects responsible for this peak disappear at annealing at the temperature near $1273 \mathrm{~K}$. These defects were suggested to be small vacancy clusters. Peak $630 \mathrm{~K}$ was attributed to release from voids. This peak (and void contribution) increase with the ion damage fluence. This peak and voids contribution increase also during annealing of the irradiated sample at high temperature $(1750 \mathrm{~K})$ due to agglomeration of small vacancy clusters and vacancies. These voids are not annealed at $1750 \mathrm{~K}$. FIB cross-section of irradiated area showed, that there are numerous voids with the dimension of about $20 \mathrm{~nm}$ in a near surface region within $500 \mathrm{~nm}$; there are no voids outside this regions as well as in the as received sample. 
This work was partly supported by a joint Grant \#07-08-92280 from the Impuls- und Vernetzungsfond der Helmholtz Gemeinschaft, Russian Foundation for Basic Researches and by Contracts $\Pi 312$ and $\Pi 1180$ with the Russian Ministry of Education and Science.

\section{References}

[1] 1, Rion A. Causey and Thomas J. Venhaus, Phys. Scr. T94, 9-15, 2001

[2] C.H.Skinner, A.A.Haasz, V.Kh.Alimov, et al, Fusion Science and Technology, 2008, v.54, p.891-945

[3] A.A. Haasz, M. Poon, J.W. Davis, J. Nucl. Mater. 266-269 (1999) 520-525

[4] H. Eleveld, A. van Veen, J. Nucl. Mater. 212-215 (1994) 1421-1425

[5] M. Poon, A.A. Haasz, J.W. Davis, J. Nucl. Mater. 374 (2008) 390-402

[6] A. Van Veen, H.A. Filius, J. de Vries, K.R. Bijkerk, G.J. Rozing, and D. Segers, J. Nucl. Mater. 155-157 (1988) 1113-1117

[7] A.A. Haasz, J.W. Davis, M. Poon, R.G. Macaulay-Newcombe, J. Nucl. Mater. 258263 (1998) 889-895

[8] H. Eleveld and A. van Veen, J. Nucl. Mater. 191-194 (1992) 433-438

[9] M. Poon, A.A. Haasz, J.W. Davis, J. Nucl. Mater. 374 (2008) 390-402

[10] A.D. Quastel, J.W. Davis, A.A. Haasz, R.G. Macaulay-Newcombe, J. Nucl. Mater. 359 (2006) 8-16

[11] A. A. Rusinov, Yu. M. Gasparyan, S. F. Perelygin, A. A. Pisarev, S. O. Stepanov, and N. N. Trifonov, Instruments and Experimental Techniques, 2009, Vol. 52, No. 6, pp. $871-876$

[12] Yu. Gasparyan, M. Rasinski, M. Mayer, A. Pisarev, J. Roth, Deuterium ion-driven permeation and bulk retention in tungsten, J. Nucl. Mater. in press 


\section{Figure captions}

Fig. 1

TDS of $\mathrm{D}_{2}$ from $\mathrm{W}$ sample, irradiated by various fluences from $2 \times 10^{16}$ to $2 \times 10^{18} \mathrm{D} / \mathrm{cm}^{2}$.All TDS in this figure were measured to $1750 \mathrm{~K}$.

Fig. 2

Series of probe $\mathrm{D}_{2}$ TDS made one after another with the sample, pre-irradiated by $6 \times 10^{17} \mathrm{D} / \mathrm{cm}^{2}$. Arrows indicate the sequence of probe implantations. Before the first probe TDS (a) and in every probe TDS, the temperature was raised to $1750 \mathrm{~K}$ and then decreased fast down to room temperature. Before the last probe TDS (b) the sample was annealed at $1750 \mathrm{~K}$ for 1 hour.

Fig. 3

A sequence (a-f) of probe TDS of $\mathrm{D}_{2}$ from a recrystallized sample, pre-irradiated by $6 \times 10^{17} \mathrm{D} / \mathrm{cm}^{2}$ and then heated to $873 \mathrm{~K}$ to remove trapped deuterium. Each TDS from (a) to (g) was made to the maximum temperatures $873,1073,1273,1473,1623,1750$, and $1750 \mathrm{~K}$, respectively. The sample was kept for 30 min after (a-e) and 1 hour after (f). Thus, the sample was kept for $1 \mathrm{~min}$ at $873 \mathrm{~K}$ before probe implantation in (a), for $30 \mathrm{~min}$ at 873, 1073, 1273, 1473 , and $1623 \mathrm{~K}$ before probe implantations (b-f), and for 1 hour at $1750 \mathrm{~K}$ before probe implantation $(\mathrm{g})$.

Fig. 4

Series of probe TDS of $\mathrm{D}_{2}$ from non-annealed and non-damaged sample: TDS were made to maximal temperatures $\mathrm{T}_{\max }=900,1090,1500,1750,1750 \mathrm{~K}$ in runs (a-e). The sample before the first probe implantations (a) was not heated. Before probe implantations $(b \div d)-$ the sample was heated at $\mathrm{T}_{\max }=900,1090,1500 \mathrm{~K}$ for $30 \mathrm{~min}$. Before probe implantation (e) the sample was heated at $1750 \mathrm{~K}$ for 1 hour.

Fig. 5

Series of probe TDS of $\mathrm{D}_{2}$ from the sample with various integral irradiation fluence $\mathrm{F}_{\max }=2 \times 10^{16}, 6 \times 10^{17}, 1 \times 10^{18}, 5 \times 10^{18} \mathrm{D} / \mathrm{cm}^{2}$.

Fig. 6 
FIB cross-section of irradiated area of the sample, irradiated up to maximal fluence of $5 \times 10^{18} \mathrm{D} / \mathrm{cm}^{2}$. Some voids are showed by a contour. The sample is coated by a carbon film (black strip), that is a standard procedure to make a cross-section. 


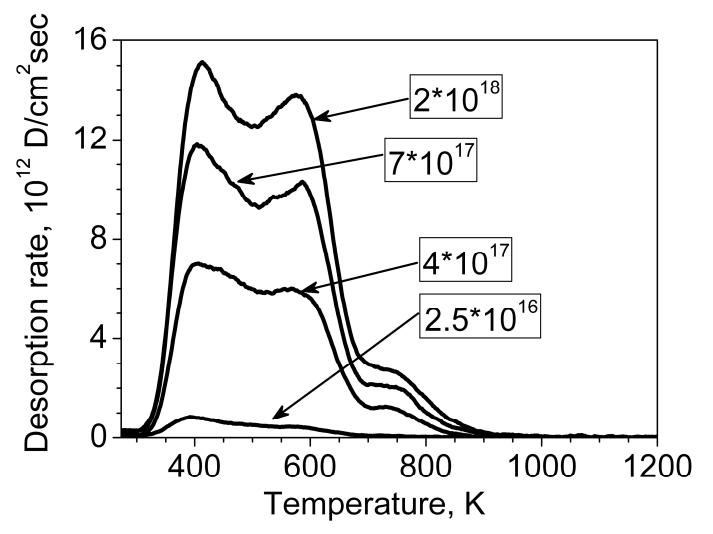

Fig. 1

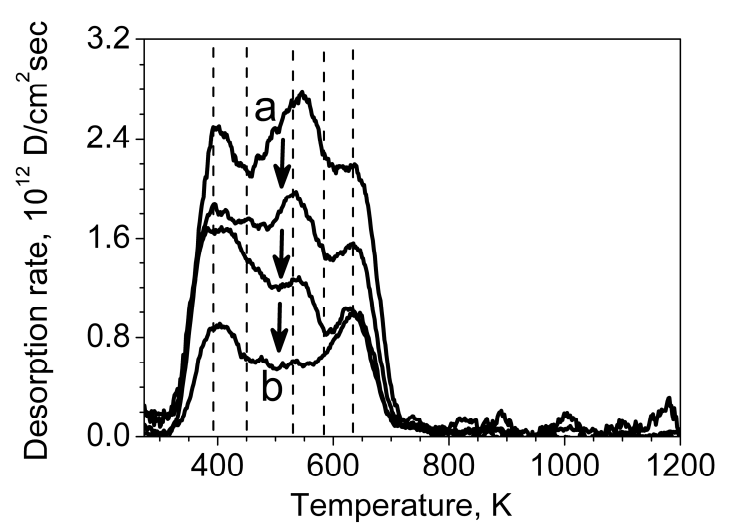

Fig. 2

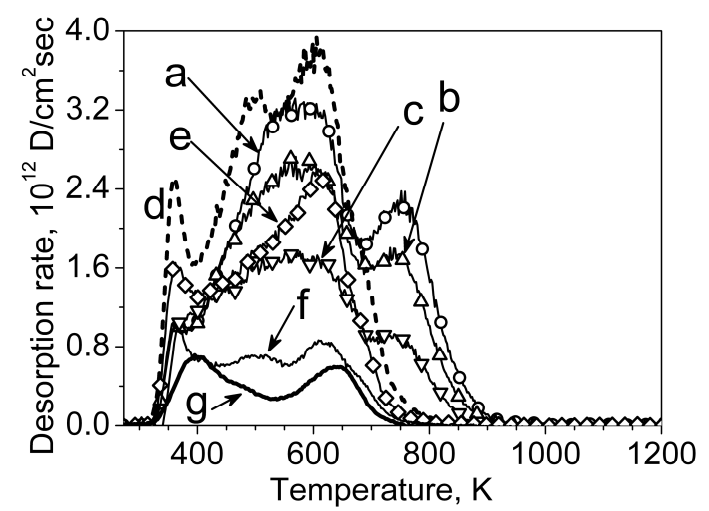

Fig. 3 


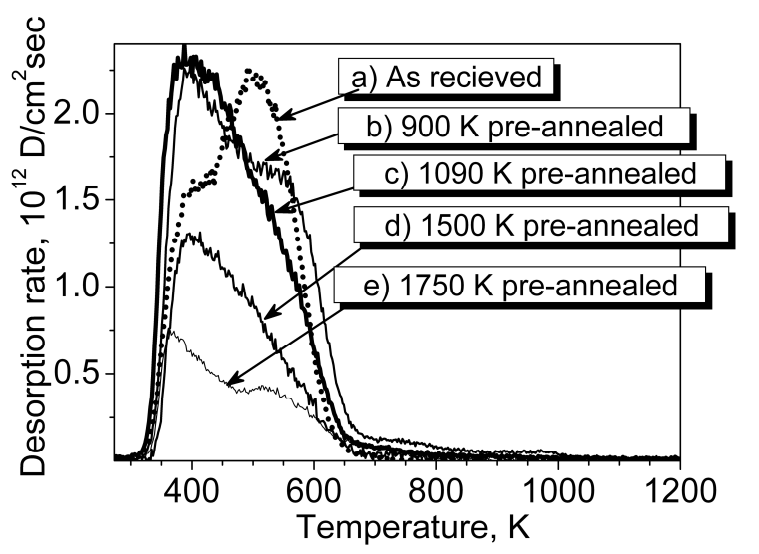

Fig. 4

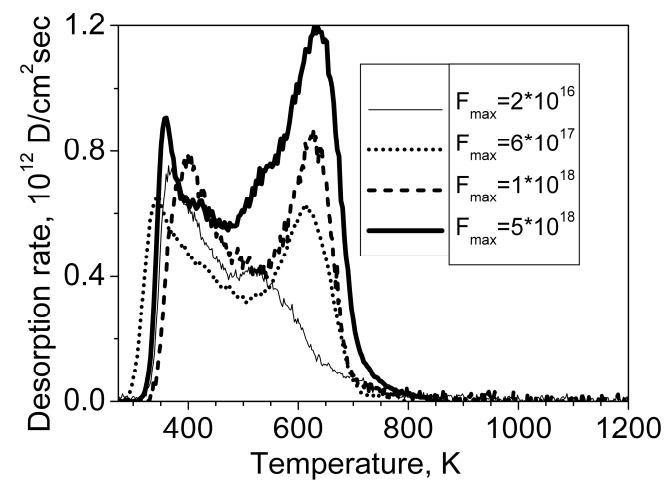

Fig. 5

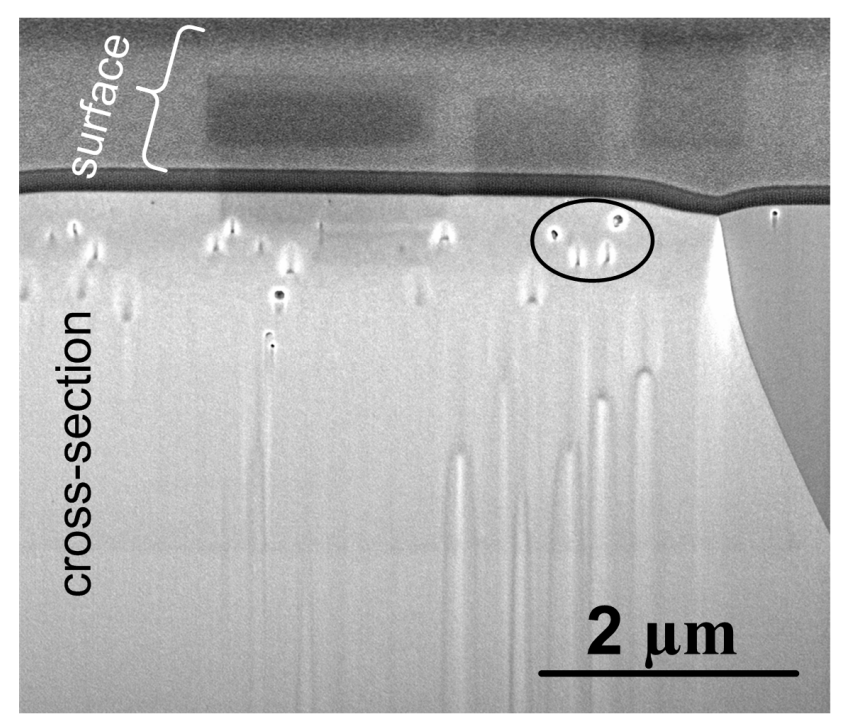

Fig. 6 